\title{
Extensive Plexiform Neurofibroma in a Premature Neonate
}

Aditya Joshi, MD, MPH; Moira Lancelot, MD, FAAP; Nandita R. Bhattacharjee, MD;

Sruthi Polavarapu, MD; Efstathios Beltaos, MD, and Babatunde Sobowale, MD, FAAP

\begin{abstract}
We describe a premature neonate with an extensive plexiform neurofibroma. Prenatal ultrasound at 32 weeks of gestation was normal. Postnatal examination was significant for a palpable left neck mass. Magnetic resonance imaging (MRI) of the head demonstrated a mass involving the left cavernous sinus with spreading to the left orbital region. MRI of the neck was positive for extensive adenopathy, left more than right, with extension into the deep face region and infratemporal fossa on the left side. MRI of the chest, abdomen, and pelvis demonstrated a mass extending from the superior mediastinum to the left pelvic retroperitoneal region, including the mesenteric vasculature and spinal canal at multiple levels with compression of the spinal cord. Biopsy of the left neck mass confirmed for plexiform neurofibroma. A careful search of the literature revealed no previous report of such an extensive neurofibroma in a premature neonate. Surgical decompression in this premature neonate was not possible because of the extensive nature of the disease; it is known that neurofibroma is non-respondent to chemotherapy and radiotherapy. Therefore, alternative treatment is needed to improve the outcome.
\end{abstract}

Keywords: Plexiform neurofibroma; Premature neonate; Treatment.

Corresponding Author:

Babatunde Sobowale MD, FAAP

Department of Pediatrics

Marshfield Clinic/Ministry Saint Joseph's

Children Hospital

Marshfield, Wisconsin 54449 USA

Tel: (7I5) 387-17/3

Email: Sobowale.babatunde@marshfieldclinic.org

Received: January 22, 2014

Ist Revision: March 14, 2014

2nd Revision: March 28, 2014

Accepted: April 3, 2014

doi: $10.3121 / \mathrm{cmr} .2014 .1224$
$\mathrm{N}$ eurofibromatosis 1, also known as Von Recklinghausen's disease, is a

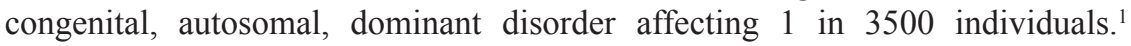
Neurofibromatosis 1 has a germ line mutation in the NF1 tumor suppression gene. ${ }^{2}$ There are various subtypes of neurofibromas including focal cutaneous, diffuse cutaneous, subcutaneous, deep nodular, and diffuse plexiform. Plexiform neurofibromas develop in about half of the individuals with neurofibromatosis $1,3,4$ and are the major cause of morbidity and disfigurement. This particular variant can also progress to malignant peripheral nerve sheath tumor (MPNST), one of the leading causes of death among patients with neurofibromatosis $1 .^{3}$

\section{Case Report}

A 2-day old male neonate with a 35-5/7 week gestation and birth weight of 2664 grams was referred to our institution for evaluation and management of a mass on left side of his neck. Labor and delivery was complicated by pregnancyinduced hypertension, premature rupture of membranes, and nonreassuring fetal heart rate. Delivery was via an emergent cesarean section. The Apgar scores were 2,6 , and 8 at 1,5 , and 10 minutes, respectively. There was no known family history suggestive of neurofibromatosis 1 . Prenatal ultrasound performed at 32 weeks of gestation was normal. 


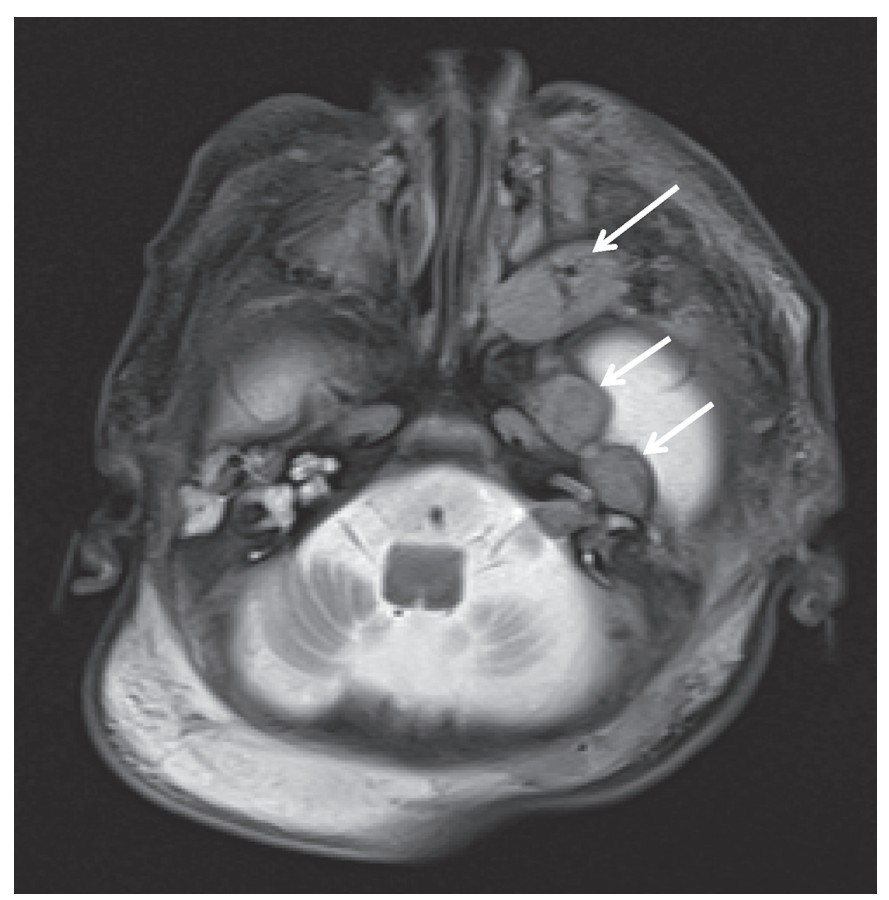

Figure 1: Magnetic resonance imaging T2 TSE Axial FS of the head. The mass lesion extends down the foramen ovale and foramen rotundum on the left side. Shown is perineural spread extending from the cavernous sinus into the orbit region with mass effect seen within the posterior half of the orbital region, intraconally and extraconally, encasing the optic nerve and the extraocularmuscles. Extension into the widened pterygopalatine fossa is noted.

On physical examination, there was a firm irregular mass in the left upper neck, $3 \mathrm{~cm} \times 3 \mathrm{~cm}$ in size with multiple lobulations involving the sternocleidomastoid muscle with normal overlying skin. No café-au-lait spots were noticed. Head was normocephalic with soft anterior fontanel. The left ear appeared larger, and the right ear had a fold in the ear lobe. Neurologically, the baby had spontaneous movement, tone was diminished bilaterally, and suck was present but poor. Fifth toes were over-riding bilaterally. He was having positional desaturation and was intubated. $\mathrm{He}$ was subsequently extubated and was put on oxygen via nasal cannula and continuous positive airway pressure.

Magnetic resonance imaging (MRI) of the head (figure 1) demonstrated a mass involving the left cavernous sinus and spreading to the left orbital region. There was hemorrhage noted in the periventricular white matter of left lateral ventricle extending from body to the posterior horn. MRI of the neck (figure 2) was positive for extensive adenopathy, left more than right, with extension into the deep face region and infratemporal fossa on the left side. The left internal carotid artery was displaced laterally and was narrowed throughout its course in the neck to the skull base. MRI of the chest, abdomen, and pelvis (figure 3 ) demonstrated a mass extending from the mediastinum to the left pelvic retroperitoneal region, including the mesenteric vasculature and spinal canal at multiple levels with compression of the spinal cord.
Karyotype was normal $46 \mathrm{XY}$. Pathology obtained from the left deep neck mass confirmed plexiform neurofibroma (figure 4). NF1 genetic testing was positive. Due to the extensive nature of the tumor in our neonate, surgery was not a viable option. The baby died after 3 weeks of conservative management.

\section{Discussion}

Plexiform neurofibromas arise from multiple nerve fascicles. They tend to grow along the length of nerve and extend into surrounding structures including skin, fascia, muscle, bone, and internal organs. Histologically, plexiform neurofibromas are characterized by an increase in the endoneurial matrix and proliferation of Schwann cells. ${ }^{5}$ Most plexiform neurofibromas are diagnosed in childhood, based on the National Institute of Health (NIH) diagnostic criteria. ${ }^{6}$ The NIH diagnostic criteria is highly sensitive and specific for the adult population, but only about half the children with NF1 and an unknown family history meet the NIH criteria by 1 year of age. ${ }^{7}$ This makes the diagnosis of neurofibroma more challenging in a patient like ours.

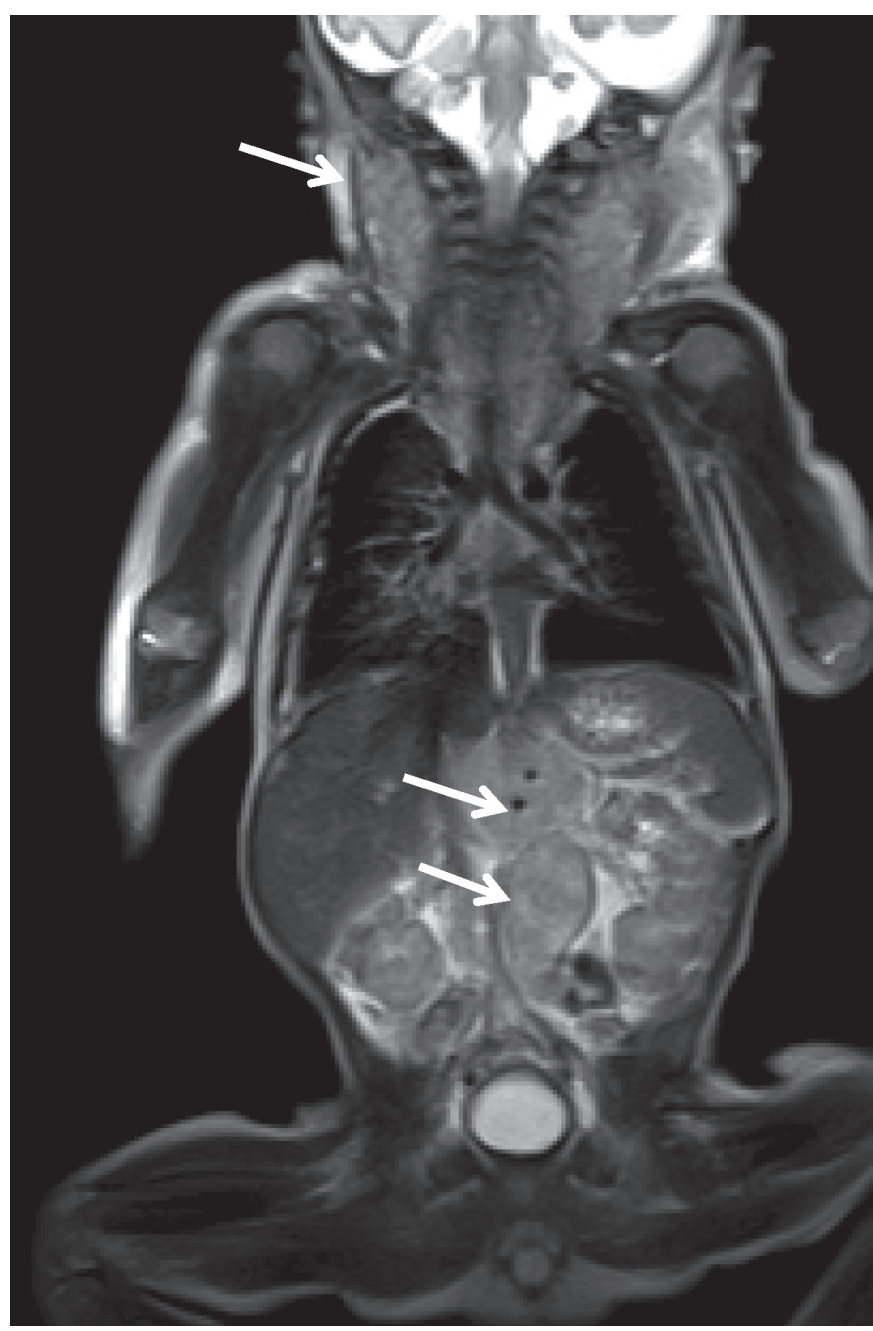

Figure 2: Magnetic resonance imaging T2 HASTE Coronal of the neck. In the neck there are large retropharyngeal lymph nodes and in the abdomen soft tissue wraps around the celiac and superior mesenteric arteries, extending anteriorly into the mesenteric root. 


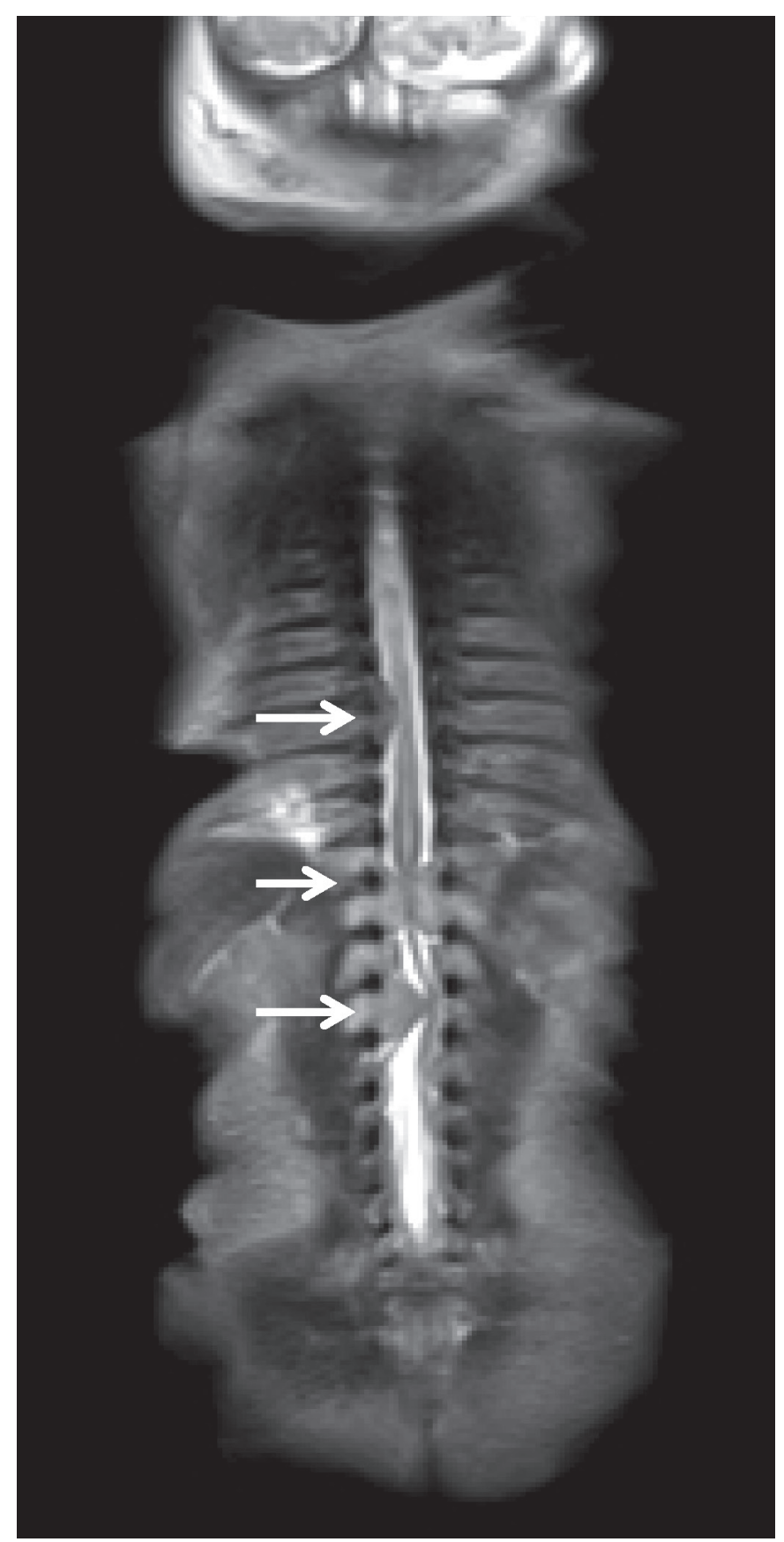

Figure 3: Magnetic resonance imaging T2 HASTE Coronal of the chest, abdomen and pelvis. Multiple soft tissue densities encroach into the spinal canal and impinge upon the spinal cord.

There are only a few reports in the literature of plexiform neurofibromas in the neonatal period. Mersal et $\mathrm{al}^{8}$ reported a 15-day-old neonate with a neck mass, causing airway and esophageal obstruction. Unlike our case, the baby also had café-au-lait spots. MRI revealed extension of the neck mass into the skull base and floor of the middle cranial fossa. Biopsy confirmed plexiform neurofibroma. The baby was managed with partial decompression of the tumor. ${ }^{8}$ Our case, however, was different because our patient was a premature neonate with severe extension of the tumor with impingement on the vital structures including the brain, spinal cord, airways, and blood vessels. We conducted a careful literature search and did not find any reports of a premature neonate with an extensive plexiform neurofibroma.

Waggoner et $\mathrm{al}^{9}$ did one of the largest retrospective studies of plexiform neurofibroma patients and their symptoms, in which they studied 68 NF1 patients with plexiform neurofibroma. They reported six patients with plexiform neurofibroma at birth and three patients with plexiform neurofibroma between birth and 6 months of age. In about $44 \%$, the tumor was localized to trunk, $38 \%$ to limbs, and $18 \%$ head and neck. No extensive tumor, as found in our patient, was reported from the cohort. Most patients in the cohort were managed surgically (53\%), with the most common reason for performing surgery being medical complications related to the tumor. ${ }^{9}$

Prenatal ultrasound in our patient at 32 weeks of gestation was reported normal. To our knowledge, diagnosis of the plexiform neurofibroma through prenatal ultrasound has not been previously reported. There have been few case reports with an abnormal perinatal ultrasound that revealed polyhydramnios, macrocephaly, and hydrocephalus; ${ }^{10}$ however, because of the nonspecific nature of the findings, prenatal diagnosis of plexiform neurofibromas via ultrasound is not possible. Although MRI is the imaging modality of choice in the postnatal period, the typical hyperintense MR lesions have not been demonstrated prenatally because of immature myelination. ${ }^{6}$

There is no specific treatment guideline for plexiform neurofibromas. They are highly refractory to radiotherapy and chemotherapy because of the slow growing nature of the tumor. $^{11}$ In a phase II trial of imatinib mesylate for plexiform neurofibromas in patients aged 3 to 52 years (median age 11 years), 23 patients received imatinib mesylate for at least 6 months; 6 patients $(26 \%, 95 \%$ CI $10-48)$ had a $20 \%$ or more decrease in the volume of one or more plexiform tumors, with a maximum of $38 \%$ reported in one of the tumors. ${ }^{12}$ In our case, imatinib was not ideal, because the tumor was negative for c-kit. A Phase II trial of sirolimus, which blocks the activity of a protein mTOR involved in cell growth, has been recently completed. Other clinical trials that include chemotherapeutic drugs, anti-angiogenesis drugs, and drugs that modulate the presumed genetic underpinnings are underway. ${ }^{13}$

Surgery still remains the main stay of treatment. ${ }^{14}$ However, surgical management is very challenging due to the diffuse infiltrative nature of the disease that impedes complete surgical resection. There is a high rate of tumor regrowth and a high risk of neurological deficit after tumor resection. In a retrospective review conducted at Children's Hospital of Philadelphia, of 121 patients who underwent resection, only $54 \%$ were free of tumor recurrence at the 10 year follow up. ${ }^{147}$ The indications for surgical intervention include cosmesis, 


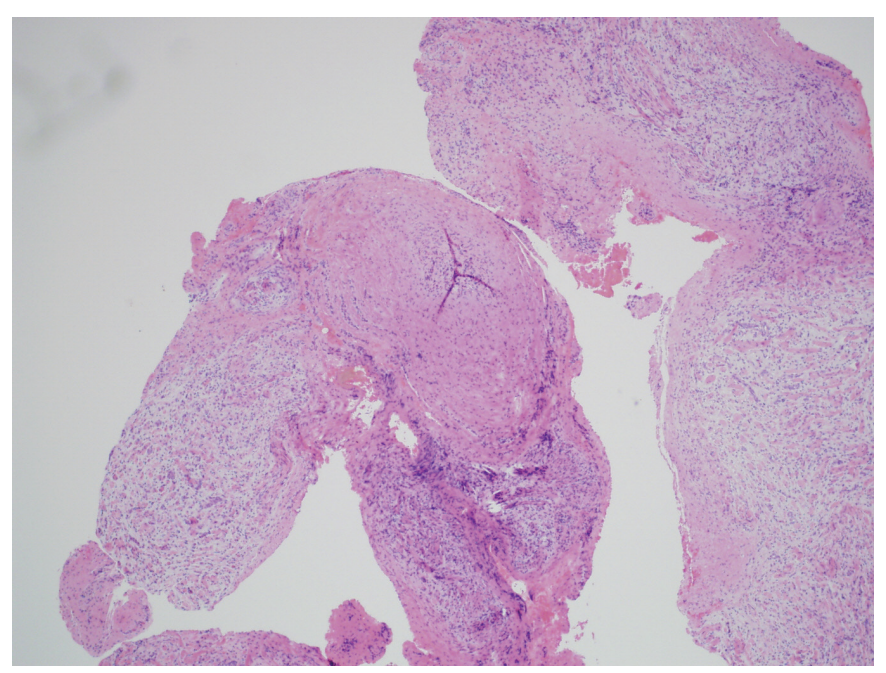

Figure 4: Hematoxylin and eosin stain showing multiple expanded nerve branches consisting of dispersed cells with often comma shaped nuclei (Schwann cells), fibroblasts, and thick collagen fibers consistent with plexiform neurofibroma. S-100 and neurofilament immunostain confirmed the presence of Schwann cells and axons.

intractable pain, neurological deficit, suspected malignant transformation, and compressive symptoms. ${ }^{15}$ Surgery was not an option because of the extensive nature of the disease that involved vital structures including the internal carotid and celiac arteries with impingement of the airway, spinal cord, and extension into brain in this premature neonate.

This is the first case of an extensive plexiform neurofibroma to be reported in a premature neonate. Family history was negative for neurofibromatosis, therefore it is possible that this was a de novo mutation. Antenatal ultrasound was not effective for diagnosing plexiform neurofibromas and surgical resection was not possible. The clinical trials for medical management have not been promising so far. Therefore, alternative treatment is needed to improve outcome.

\section{Acknowledgements}

The authors thank the Office of Scientific Writing and Publication at the Marshfield Clinic Research Foundation for assistance with the preparation of this manuscript.

\section{References}

1. Rasmussen SA, Friedman JM. NF1 gene and neurofibromatosis 1. Am J Epidemiol 2000;151:33-40.

2. Viskochil D, Buchbert AM, Xu G, Cawthon RM, Stevens J, Wolff RK, Culver M, Carey JC, Copeland NG, Jenkins NA, et al. Deletions and a translocation interrupt a cloned gene at the neurofibromatosis type 1 locus. Cell 1990;62:187-192.

3. Tucker T, Friedman JM, Friedrich RE, Wenzel R, Funsterer C, Mautner VF. Longitudinal study of neurofibromatosis 1 associated plexiform neurofibromas. J Med Genet 2009; 46:81-85.

4. Thakkar SD, Feigen U, MautnerVF. Spinal tumours in neurofibromatosis type 1: an MRI study of frequency, multiplicity and variety. Neuroradiology 1999;41:625-629.

5. Wening B. Atlas of head and neck pathology. 1st ed. Philadelphia, PA: WB Saunders; 1993.
6. McEwing RL, Joelle R, Mohlo M, Bernard JP, Hillion Y, Ville Y. Prenatal diagnosis of neurofibromatosis type 1: sonographic and MRI findings. Prenat Diagn 2006;26:1110-1114.

7. DeBella K, Poskitt K, Szudek J, Friedman JM. Use of "unidentified bright objects" on MRI for diagnosis of neurofibromatosis 1 in children. Neurology 2000; 54:1646-1651.

8. Mersal AY, Hassan AA, Alardata HA, Al-Harthi A, Avand G. Congenital neurofibromatosis in a Saudi neonate who presented with neck mass, esophageal and airway obstruction. J Clin Neonatol 2012;1:214-216.

9. Waggoner D, Towbin J, Gottesman G, Gutman D. Clinical based study of plexiform neurofibromas in neurofibromatosis 1 . American Journal of Medical Genetics 2000; 92: 132-135.

10. Drouin V, Marret S, Petitcolar J, Eurin D, Vannier JP, Fessard C, Tron P. Prenatal ultrasound abnormalities in a patient with generalized neurofibromatosis type 1 . Neuropediatrics 1997;28:120-121.

11. Lantieri L, Meningaud JP, Grimbert P, Bellivier F, Lefaucheur JP, Ortonne N, Benjoar MD, Lang P, Wolkenstein P. Repair of the lower and middle parts of the face by composite tissue allotransplantation in a patient with massive plexiform neurofibroma: a 1-year follow-up study. Lancet 2008;372:639-645

12. Robertson KA, Nalepa G, Yang FC, Bowers DC, Ho CY, Hutchins GD, Croop JM, Vik TA, Denne SC, Parada LF, Hingtgen CM, Walsh LE, Yu M, Pradhan KR, EdwardsBrown MK, Cohen MD, Fletcher JW, Travers JB, Staser KW, Lee MW, Sherman MR, Davis CJ, Miller LC, Ingram DA, Clapp DW. Imatinib mesylate for plexiform neurofibromas in patients with neurofibromatosis type 1: a phase 2 trial. Lancet Oncol 2012;13:1218-1224.

13. Ardern-Holmes SL, North KN. Treatment for plexiform neurofibromas in patients with NF1. Lancet Oncol 2012;13:1175-1176.

14. Needle MN, Cnaan A, Dattilo J, Chatten J, Phillips PC, Shochat S, Sutton LN, Vaughan SN, Zackai EH, Zhao H, Molloy PT. Prognostic signs in the surgical management of plexiform neurofibroma: the Children's Hospital of Philadelphia experience, 1974-1994. J Pediatr 1997; 131: 678-682.

15. Bhattacharyya AK, Perrin R, Guha A. Peripheral nerve tumors: management strategies and molecular insights. J Neurooncol 2004;69:335-349.

\section{Author Affiliations}

Aditya Joshi, MD, MPH*; Moira Lancelot, MD, $F A A P^{\dagger}$,

Nandita R. Bhattacharjee, MD"

Sruthi Polavarapu*, MD, Efstathios Beltaos, $M D^{\S}$, Babatunde Sobowale, MD, FAAP

*Department of Pediatrics, Marshfield Clinic/Ministry Saint Joseph's Children Hospital, Marshfield, Wisconsin, USA.

'Department of Pediatric Oncology, Marshfield Clinicl Ministry Saint Joseph's Children Hospital, Marshfield, Wisconsin, USA.

${ }^{*}$ Department of Radiology, Marshfield Clinic, Marshfield, Wisconsin, USA.

${ }^{\S}$ Department of Lab-Pathology, Marshfield Clinic, Marshfield, Wisconsin, USA.

'Department of Pediatrics/Neonatology, Marshfield Clinic/ Ministry Saint Joseph's Children Hospital, Marshfield, Wisconsin, USA. 